\title{
The effect of levocetirizine and montelukast on clinical symptoms, serum level and skin expression of COX-1 and COX-2 enzymes in patients suffering from chronic autoimmune urticaria - a pilot study
}

\author{
Paulina Korczyńska-Krawczyk ${ }^{1}$, Izabela Kupryś-Lipińska ${ }^{1}$, Maciej Kupczyk ${ }^{1}$, Małgorzata Wągrowska-Danilewicz², \\ Janusz Szemraj ${ }^{3}$, Wojciech Bienias ${ }^{4}$, Joanna Narbutt ${ }^{4}$, Janusz Śmigielski ${ }^{5}$, Piotr Kuna ${ }^{1}$
}



Adv Dermatol Allergol 2020; XXXVII (1): 73-80 DOI: https://doi.org/10.5114/ada.2018.79731

\begin{abstract}
Introduction: Chronic autoimmune urticaria (CAU) lasts over 6 weeks and is characterized by circulating IgE autoantibodies or IgG against IgE or IgE receptor.

Aim: To assess the clinical, laboratory and histological effects of 4-week levocetirizine and montelukast therapy in patients suffering from CAU.

Material and methods: Of 296 tested patients with chronic urticaria 40 had a positive ASST test. Only 17 (16 female/1 male; medium age: 44 years) fulfilled all study inclusion/exclusion criteria. The study was designed as an open, randomized trial with two arms: levocetirizine or montelukast treatment for 4 weeks following a 2-week wash-out period. All participants completed urticaria activity score (UAS) and visual analogue scale (VAS) questionnaires before and after both therapies. Blood samples and skin bioptats were obtained before and after treatment to evaluate COX-1 and COX-2 serum concentrations and skin expression.

Results: Clinical response to therapy measured with the UAS and VAS was better in the levocetirizine group. Both drugs caused a significant decrease in COX-1 and COX-2 serum level. COX-1 and COX-2 expression in epidermal and dermal inflammatory infiltration did not change significantly in either study group, but a significant decrease of COX-1 expression was observed when the groups were combined for analysis, and the decrease in COX-2 expression in the epidermis was of borderline significance.

Conclusions: The effectiveness of levocetirizine and montelukast in treating CAU may be partly related to the reduction of COX-1 and COX-2 serum level and tissue expression, but further studies on a larger group of patients are needed to support this observation.
\end{abstract}

Key words: chronic urticaria, cyclooxygenase, levocetirizine, montelukast.

\section{Introduction}

Chronic urticaria (CU) is a phenotypically heterogeneous syndrome characterized by the continuous or recurring presence of urticarial wheals over 6 weeks. Urticaria is typically classified as spontaneous or physical based on the appearance of symptoms, these being spontaneous or triggered by physical factors. In Poland, $0.6 \%$ of the population ( $95 \% \mathrm{Cl}$ : $0.4-0.8 \%$ ) suffered from chronic spontaneous urticaria, with women being affected more often than men $(0.8 \%$ vs. $0.3 \% ; p<0.05)[1]$. As many as $50 \%$ of chronic spontaneous urticaria (CSU) patients have an autoimmune etiology [2].

Address for correspondence: Paulina Korczyńska-Krawczyk MD, Department of Internal Medicine, Asthma and Allergy, Norbert Barlicki Memorial University Hospital No. 1, Medical University of Lodz, 22 Kopcińskiego St, 90-153 Lodz, Poland, phone: +48 508 308443 , fax: +48 4267769 51, e-mail: paulina.korkraw@gmail.com Received: 1.07.2018, accepted: 8.10.2018. 
Autoantibodies (in the IgG class) against IgE or $\alpha$ subunit of IgE receptor (Fc\&Rl $\alpha$ ) may be detected in chronic autoimmune urticaria, as well as autoantibodies in the E class against autoantigens $[3,4]$. The autoantibodies cause the degranulation of dermal mast cells and basophils, followed, within minutes or hours, by the release of numerous inflammatory mediators including histamine, serotonin, various interleukins and tumor necrosis factor $\alpha$ (TNF- $\alpha$ ). They also stimulate the release of leukotrienes, prostaglandins and thromboxanes, as well as other lipid mediators, which are also known to take part in the inflammatory reaction.

Histamine is the main mediator responsible for the erythema, wheals and swelling in urticaria. The binding of histamine to the $\mathrm{H} 1$ receptors on small cutaneous blood vessels causes vasopermeability and vasodilatation. It also mediates itching through stimulation of cutaneous nociceptors and the surrounding flare following antidromic stimulation of local C-fiber networks.

The cysteinyl leukotrienes may also contribute to vasopermeability and vasodilatation in urticaria but are secondary in importance to histamine. The release of preformed leukotrienes, as well as the de novo synthesis of LTC4, LTD4 and LTE4 by mast cells at the time of degranulation, and subsequently by infiltrating basophils and eosinophils, may result in the prolongation of urticaria wheals in aspirin-sensitive urticaria, autoimmune urticaria and delayed pressure urticaria [5].

Arachidonic acid from the cell membrane is used as the precursor for leukotrienes, prostanoids and thromboxanes. Its metabolism is directed by the cyclooxygenase enzyme system, known as prostaglandin-endoperoxide $\mathrm{H}$ synthase. This enzyme occurs in two isoenzyme forms: cyclooxygenase-1 (COX-1) and cyclooxygenase-2 (COX-2). COX-1 is expressed in many cell types and, under physiological conditions, is responsible for the synthesis of prostanoids. COX-1b, also known as COX-3, is a variant of COX-1 whose role in human physiology is unclear. COX-2 is induced in cells during an inflammatory reaction and is responsible for increased production of prostanoids [6].

\section{Aim}

The aim of this study was to evaluate the effect of levocetirizine and montelukast on the clinical symptoms of chronic autoimmune urticaria, as well as on the serum level and skin expression of COX-1 and COX-2 in these patients.

\section{Material and methods \\ Study group}

A group of 296 patients with chronic urticaria was screened to select those with autoimmune etiology of the disease. Forty of these patients (13.5\%) were positive for the autologous serum skin test (ASST). The method and criteria of evaluation of ASST were performed in accordance with the position paper of the EAACI [3]. Seventeen chronic autoimmune urticaria patients (16 female; 1 male) who fulfilled the inclusion/exclusion criteria (Table 1) and gave their written informed consent were included in the study.

\section{Study design}

After a wash-out period of at least 2 weeks, the patients were randomly assigned into two arms: 10 patients were included in the group receiving levocetirizine $(5 \mathrm{mg}$ / day) and 7 patients were included in the group receiving montelukast (10 mg/day). Levocetirizine (5 mg daily) or montelukast (10 mg daily) was given for 4 weeks. Compliance was enforced by checking the returned packaging. Clinical status, laboratory and histological samples were evaluated twice: once at the baseline and once at the end of the treatment phase.

\section{Clinical assessment}

At the baseline and at the end of the study, all patients completed urticaria activity score (UAS) and visual analogue scale (VAS) questionnaires to assess the clinical symptoms of the disease before and after the treatment. The number of urticarial wheals and the intensity of itching were evaluated from 0 to 6 points with the UAS, where 6 points was the maximum daily disease intensity. The level of pruritus and severity of urticarial wheals were subjectively evaluated using the VAS questionnaire, by indicating a position along a continuous line between two end-points representing 0 and 10 points. A score of 10 points indicated maximum disease intensity.

Table 1. Inclusion and exclusion criteria

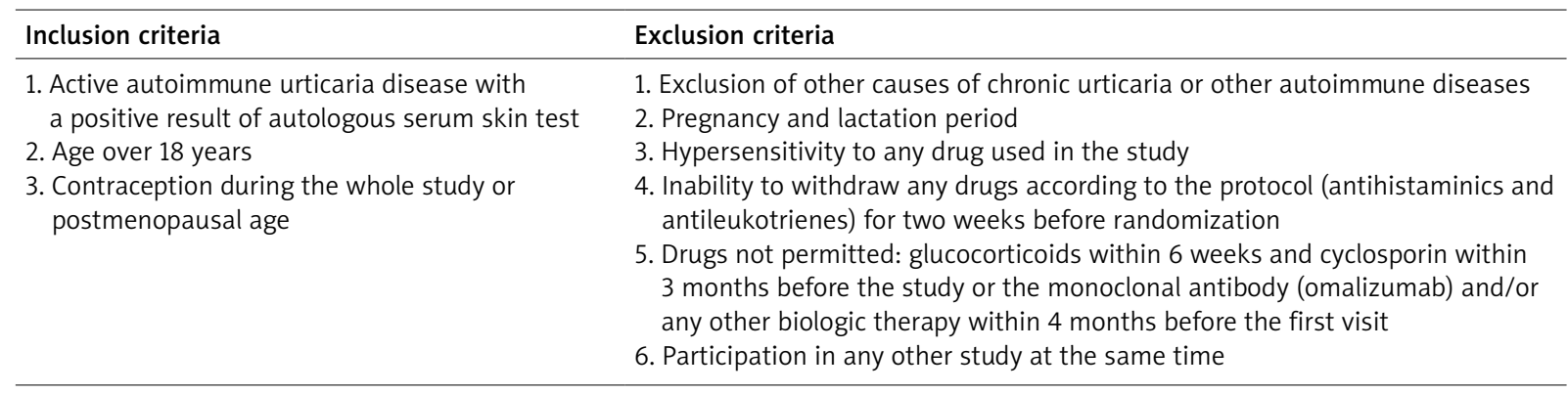




\section{Serum levels of COX-1 and COX-2}

The level of circulating COX-1 and COX-2 in the serum was measured by Enzyme-Linked Immunosorbent Assay (ELISA), with the Monoclonal Anti-Human COX-1 and COX-2 Antibodies from Human Prostaglandin $\mathrm{G} / \mathrm{H}$ synthase 1 and Human Prostaglandin G/H synthase 2 ELISA kit (EIAab). The procedure was performed and the results were calculated according to the manufacturer's recommendations.

\section{Biopsy}

The skin bioptats ( $3 \mathrm{~mm}$ punch biopsies) were obtained twice, once before and once after the treatment, to evaluate COX-1, COX-2 skin expression with immunohistochemistry. The bioptats were taken from the urticarial wheals twice and the histopathology examination was performed both before and after the treatment. Only 8 patients gave permission for a skin biopsy to be taken.

The bioptats were fixed in Bouin's solution for $24 \mathrm{~h}$. After fixation, the tissue was dehydrated in a series of alcohols, cleared in xylene and embedded in paraffin. Sections measuring $3 \mu \mathrm{m}$ were cut with a microtome (Microtome Leica RM 2245); they were then mounted on Super Frost Ultra Plus charged glass slides (Menzel Glaser, Germany), dried at $50^{\circ} \mathrm{C}$ for $1 \mathrm{~h}$ and stored at room temperature until use. On the day of the experiment, the slides were dewaxed in xylene, rehydrated in a series of alcohols and washed with distilled water. The samples were rinsed for $20 \mathrm{~min}$ at $97^{\circ} \mathrm{C}$ in Target Retrieval Solution buffer at pH 6.0 for COX-1 and pH 9.0 for COX-2.

The samples were rinsed in Tris-Buffered Saline buffer ( $\mathrm{pH}$ 7.6). To block endogenous peroxidase, the sections were incubated with peroxidase block reagent for $5 \mathrm{~min}$ at room temperature using a DakoCytomation EnVision + System, HRP (DAB) kit (Dako, Glostrup Denmark).

\section{Skin expression of COX-1 and COX-2}

Immunohistochemistry was performed to assess COX-1 and COX-2 skin expression, with Anti-COX-1 Antibody 5F6/F4 ab695 (Abcam) and Monoclonal Mouse Anti-Human COX-2 Clone CX 294 (Dako). COX-1 and COX-2 expression was evaluated in the epidermis and dermis inflammatory infiltrations.

Both cyclooxygenases were assessed semi-quantitatively at two time points, in five fields of view, using an Olympus BX41 microscope at 400× magnification. Immunoexpression was evaluated on a scale of 0-3 points, where 0 = no expression, 1 = immunoexpression up to $30 \%, 2$ = immunoexpression between $30 \%$ and $60 \%$, and 3 =immunoexpression above $60 \%$.

Monoclonal mouse anti-human COX-1 antibody (Abcam ) (1: 400 dilution) and monoclonal mouse anti human COX-2 (DakoCytomation) (1: 50 dilution) were used to assess COX-1 and COX-2 skin expression. The samples were first rinsed twice in TBS, treated with peroxide La- belled Polymer Reagent for $30 \mathrm{~min}$, and then rinsed twice more with TBS. They were then treated with diaminobenzidine (DAB) for $5 \mathrm{~min}$. The staining procedure with hematoxylin was identical for all the samples. The sections were dehydrated in a series of alcohols and cleared in xylene. Finally, the specimens were observed under an Olympus BX41 microscope.

\section{Statistical analysis}

Statistical analysis was performed using PQStat version 1.6. Patient age and duration of CSU were analyzed with the Mann-Whitney U-test. Sex, coexistence of angioedema, UAS and VAS were evaluated qualitatively. The Mann-Whitney U-test and Wilcoxon's pair test were used to analyze the results of the study. $P<0.05$ was considered statistically significant and $p<0.01$ highly statistically significant.

\section{Ethics}

This study was performed in accordance with the principles established in the Declaration of Helsinki and the International Conference on Harmonisation guidelines for Good Clinical Practice. All study documents and procedures were approved by the Regional Ethics Committee of the Medical University of Lodz (agreement number RNN/144/10/KE). All patients provided written informed consent before participation in the study.

\section{Results}

The medium age of the study group was 44 years. The medium duration of CSU in the study group was 53.65 months. Concomitant angioedema was observed in 12 of the CSU patients. None of the patients had any other skin disease or any disease with accompanying pruritus.

\section{Clinical response to levocetirizine and montelukast therapy \\ Changes in UAS}

At the end of the treatment period, the UAS scores indicated clinical improvement in $6 / 10$ patients treated with levocetirizine and 3/7 patients treated with montelukast. The remainder of the subjects were non-responders: in the levocetirizine group, 3/10 patients did not report any change in clinical symptoms and 1/10 reported exacerbation, while in the montelukast group, $1 / 7$ patients did not report any change and 3/7 patients noted aggravation of symptoms.

\section{Changes in VAS}

\section{VAS - wheals severity analysis}

Regarding the responders, for the severity of urticarial wheals evaluated by VAS, 2/10 subjects in the levoce- 
tirizine group and $3 / 7$ subjects in the montelukast group reported a reduction in the intensity of wheals.

However, in the levocetirizine group, $7 / 10$ subjects did not report any change in wheal intensity and $1 / 10$ reported aggravation. In the montelukast group, 1/7 patients did not report any change in wheal intensity and $3 / 7$ reported increased severity of wheals.

\section{VAS - itching severity analysis}

Itching severity according to the VAS analysis revealed that $5 / 10$ patients treated with levocetirizine and 2/7 treated with montelukast achieved a decrease of pruritus.

In the levocetirizine group, 4/10 did not report any change in the intensity of itching and $1 / 10$ reported aggravation. In the montelukast group, 2/7 patients did not report any changes and $3 / 7$ reported exacerbation of pruritus.

\section{Impact of levocetirizine and montelukast therapy on COX-1 and COX-2 serum levels and tissue expression}

\section{Changes in COX-1 and COX-2 serum level}

COX-1 serum level has decreased during the treatment in 10/10 levocetirizine patients and 6/7 montelukast patients. In the montelukast group, 1 patient demonstrated an increased COX-1 serum level after the treatment (Figure 1).

Lowered COX-2 serum level after the treatment was observed in 9/10 levocetirizine patients and 7/7 monte- lukast patients. One patient in the levocetirizine group presented a higher COX-2 serum level after the treatment (Figure 2).

Significant decreases in COX-1 serum level were identified after the treatment in the levocetirizine group ( $p=$ 0.0059), in the montelukast group ( $p=0.0346)$ and when both groups were combined for analysis (levocetirizine + montelukast) ( $p=0.0005)$.

Similarly, significant reductions in COX-2 serum level were observed in the levocetirizine group $(p=0.0080)$, in the montelukast group $(p=0.0225)$, and in both groups combined for analysis $(p=0.0004)$.

\section{Changes in COX - 1 and COX- 2 skin expression (Figure 3)}

\section{Changes in the epidermis}

COX-1 expression in the epidermis was reduced in $2 / 3$ subjects in the levocetirizine group and in $5 / 5$ subjects in the montelukast group. Increased COX-1 epidermis expression was observed in $1 / 3$ subjects in the levocetirizine group (Figure 4).

COX-2 epidermis expression was decreased in $2 / 3$ subjects in the levocetirizine group and in $4 / 5$ subjects in the montelukast group.

Increased COX-2 epidermis expression was observed in $1 / 3$ subjects in the levocetirizine group and in $1 / 5$ subjects in the montelukast group (Figure 5).

While a significant decrease of epidermal COX-1 expression was observed in the combined study groups ( $p=0.0209)$, no such significant difference was noted in either study group analyzed separately: in the levoce-

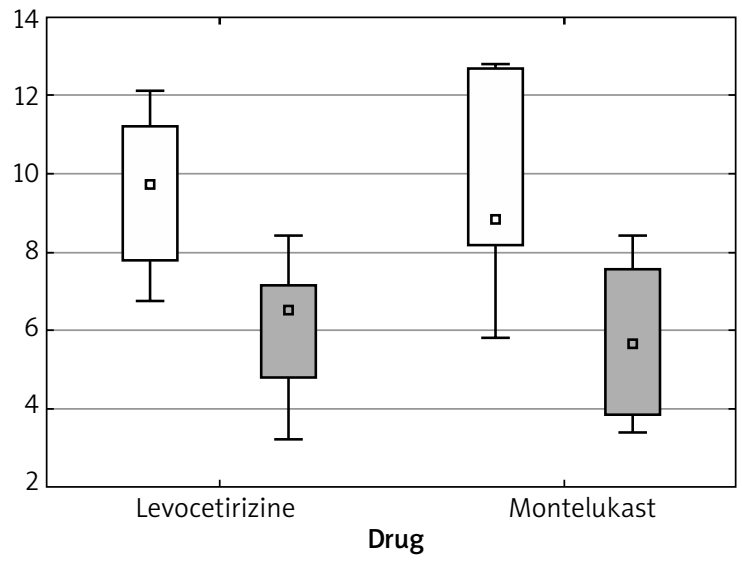

口 COX-1 serum level before the treatment

口 COX-1 serum level after the treatment

$$
\begin{aligned}
& \text { Median } \\
& \text { } 25 \%-75 \% \\
& \text { I Non-outliers range }
\end{aligned}
$$



回 COX-2 serum level before the treatment

口 COX-2 serum level after the treatment

$$
\begin{aligned}
& \text { a Median } \\
& \text { I } 25 \%-75 \% \\
& \text { I Non-outliers range }
\end{aligned}
$$

Figure 2. COX-2 serum level

Figure 1. COX-1 serum level 

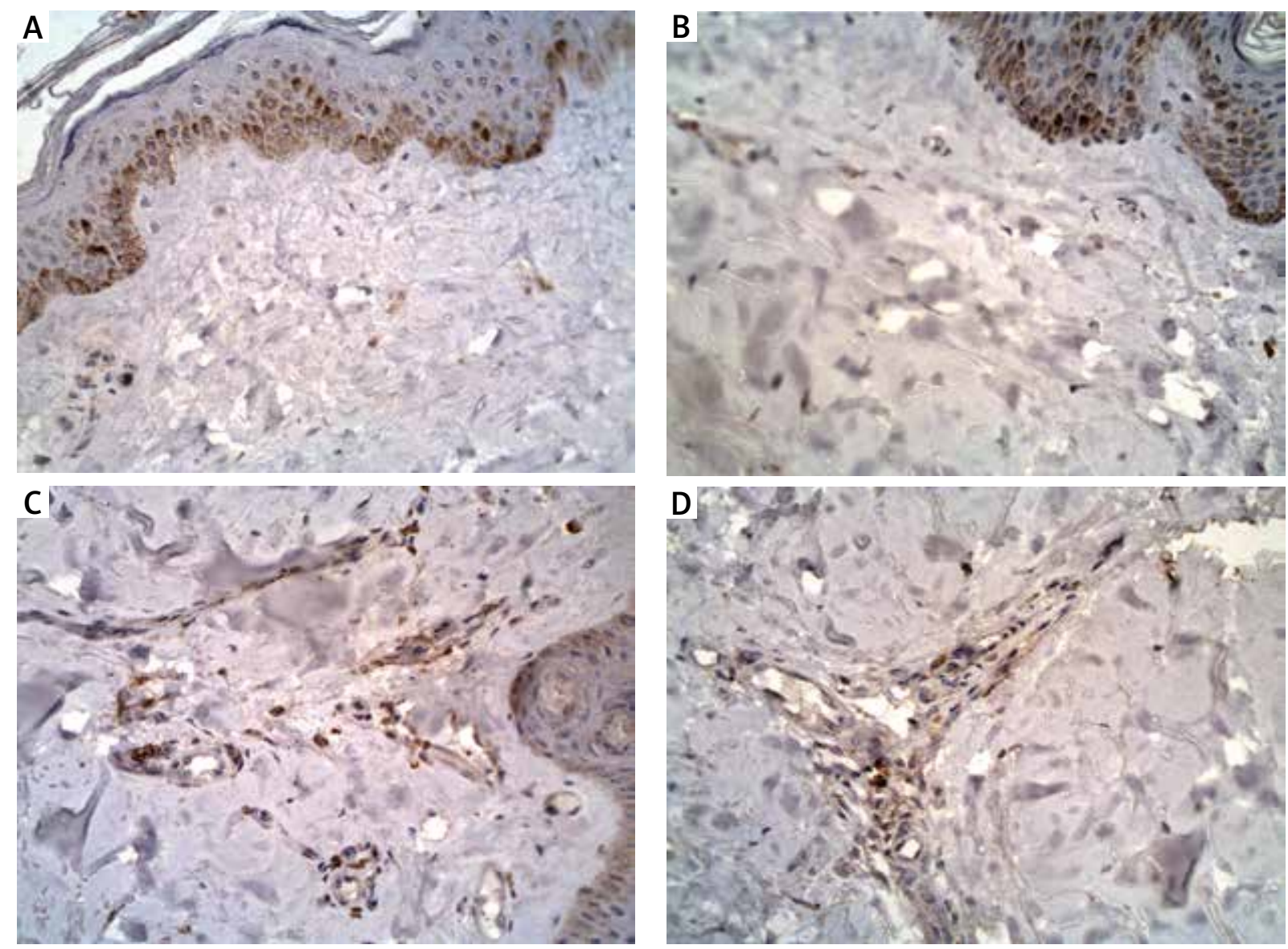

Figure 3. Staining for cyclooxygenase-1 and cyclooxygenase-2 in a specimen from a patient with autoimmune urticaria

tirizine group ( $p=0.4227$ ) or in the montelukast group $(p=0.0591)$.

No significant change in COX-2 expression in the epidermis was found for the combined study groups ( $p=$ 0.0587); however, this value is close to the significance borderline. Similarly, no significant change was observed in either study groups analyzed separately: in the levocetirizine group ( $p=0.4227$ ) or in the montelukast group $(p=0.1056)$.

\section{Changes in the dermis inflammatory infiltration}

COX-1 expression in the dermis inflammatory infiltration decreased after the treatment in $2 / 3$ subjects in the levocetirizine group and in $4 / 5$ subjects in the montelukast group. In addition, 1/3 subjects in the levocetirizine group and $1 / 5$ subjects in the montelukast group demonstrated increased COX-1 expression in the dermis inflammatory infiltration after the treatment (Figure 4).

The COX-2 expression in the dermis inflammatory infiltration was reduced in $2 / 3$ subjects in the levocetirizine group and in $4 / 5$ subjects in the montelukast group. However, COX-2 expression in the dermis inflammatory infiltration has increased after the therapy in $1 / 3$ subjects in the levocetirizine group and in $1 / 5$ subjects in the montelukast group (Figure 5).

No significant change in COX-1 expression in the dermis inflammatory infiltration was observed after the treatment in the levocetirizine group ( $p=0.4227)$ or montelukast ( $p=0.1362$ ) group assessed separately. However, a significant decrease in COX-1 expression in the dermis inflammatory infiltration was found when both study groups were combined for analysis ( $p=0.0497$ ).

No significant change in COX-2 expression in the dermis inflammatory infiltration was observed for the levocetirizine group ( $p=0.7893)$, for the montelukast group $(p=0.1775)$ or for the two groups combined for analysis $(p=0.1073)$.

\section{Adverse events}

No adverse events were associated with the levocetirizine or montelukast therapy during the study.

\section{Discussion}

Levocetirizine is a second-generation antihistamine approved for the treatment of chronic urticaria. Several 
P. Korczyńska-Krawczyk, I. Kupryś-Lipińska, M. Kupczyk, M. Wągrowska-Danilewicz, J. Szemraj, W. Bienias, J. Narbutt,

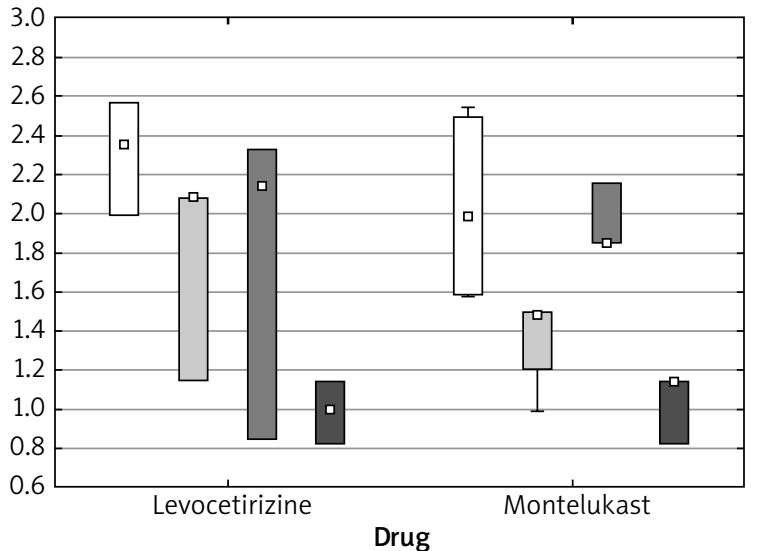

回 COX-1 expression in the epidermis before the treatment

口 COX-1 expression in the epidermis after the treatment

回 COX-1 expression in the dermis inflammatory infiltrations before the treatment

COX-1 expression in the dermis inflammatory infiltrations after the treatment

$$
\begin{aligned}
& \text { a Median } \\
& \text { } 25 \%-75 \% \\
& \text { I Non-outliers range }
\end{aligned}
$$

Figure 4. COX-1 skin expression in the epidermis and in the dermis inflammatory infiltrations before and after the treatment

randomized, controlled studies have confirmed its efficacy and safety in CU patients when compared with placebo [7] and other antihistamines such as desloratadine [8]. In the present study, 6/10 patients receiving levocetirizine reported an improvement in urticarial symptoms assessed with UAS, while 2/10 indicated reduction of wheal intensity and 5/10 noted a reduction in itching severity according to the VAS.

Montelukast is an anti-leukotriene medication approved for asthma therapy; however, some data suggest that montelukast may also be effective as an add-on therapy in chronic resistant urticaria. Erbagci [9] found that montelukast in monotherapy significantly reduces the symptoms of chronic urticaria when assessed with UAS in comparison to placebo $(p<0.001)$. However, Di Lorenzo et al. did not obtain such positive results 27 out of 40 patients treated with montelukast in monotherapy discontinued the study due to increased urticaria symptoms [10].

In our study 3 patients out of 7 treated with montelukast reported improvement in urticaria symptoms, according to the UAS; another 3 subjects reported lower severity of wheals, according to the VAS; and only 2 reported decrease of pruritus, measured with the VAS. These lower rates of significant clinical improvement in both study groups may be related to the presence of mild symptoms at the beginning of the study, the short period of treatment or limited number of subjects.



口 COX-2 expression in the epidermis before the treatment

回 COX-2 expression in the epidermis after the treatment

回 COX-2 expression in the dermis inflammatory infiltrations before the treatment

COX-2 expression in the dermis inflammatory infiltrations after the treatment

$$
\begin{aligned}
& \text { - Median } \\
& \text { } 25 \%-75 \% \\
& \text { I Non-outliers range }
\end{aligned}
$$

Figure 5. COX-2 skin expression in the epidermis and in the dermis inflammatory infiltrations before and after the treatment

A significant reduction in COX-1 and COX-2 serum level was observed after the therapy in both study groups assessed separately. A significant decrease in COX-1 skin expression was noticed both in the epidermis and in the dermis after the treatment, assessed in combined groups. COX-2 skin expression both in the epidermis and in the dermis was found to be decreased after the therapy in most of the studied patients in both groups. This is the first example of such an observation, and it seems to be necessary to confirm these findings on a larger group of patients.

Levocetirizine typically acts through the inhibition of $\mathrm{H} 1$ receptor activity. Some studies suggest that levocetirizine may also have anti-inflammatory properties. Thomson et al. [11] report that levocetirizine and cetirizine inhibit eotaxin-induced eosinophil transendothelial migration through both dermal and lung microvascular endothelial cells in vitro. Ciprandi et al. note that levocetirizine significantly reduces the number of neutrophils, eosinophils and IL-8 levels in the nasal secretion of patients with seasonal allergic rhinitis in comparison with desloratadine or placebo [12].

Only a few publications discuss the anti-inflammatory effect of levocetirizine in chronic spontaneous urticaria. Caproni et al. [13] report a significant reduction in the levels of circulating P-selectin and E-selectin adhesion molecules and a clinical improvement in chronic urticaria patients after levocetirizine treatment. 
The authors speculate that a reduction in cell adhesion molecule expression on endothelial cells, following levocetirizine treatment, may be associated with the inhibitory effect on neutrophil rolling and extravasation in inflamed skin.

Montelukast inhibits the action of the leukotrienes C4, D4 and E4 on the cysteinyl leukotriene receptor CysLT1. Although anti-inflammatory properties of montelukast in chronic urticaria remain unknown, its anti-inflammatory effects have been studied in asthma patients with nasal polyposis. Schäper et al. [14] report a significant reduction in various inflammatory mediators, including SP, NKA, cysLTS, ECP and albumin, in nasal lavage fluid after montelukast treatment and a reduction in eosinophils in nasal smears and peripheral blood after montelukast therapy $(p<0.01)$ compared to placebo.

The role of COX metabolites in urticaria is still under investigation. In addition to histamine, cutaneous mast cells release numerous vasoactive and pro-inflammatory mediators including prostaglandin D2 (PGD2), synthesized through the cyclooxygenase pathway, and leukotriene C4 (LTC4), synthesized through the lipoxygenase pathway. These prostanoids are responsible for, among others; for oedema, fever and pain.

Aspirin and other non-selective NSAIDs may activate mast cells indirectly by inhibiting the formation of prostaglandin E2 (PGE2) via cyclooxygenase (COX), which has an inhibitory effect on immunological mast cell activation. The inhibition of the COX pathway results in overproduction of cysteinyl leukotrienes [5].

Pacor et al. found that patients with chronic urticaria and intolerance to food additives and/or acetylsalicylic acid, which is related to overproduction of cysteinyl leukotrienes, demonstrate a good response to montelukast, which supports the pathogenic role of cysteinyl leukotrienes in this urticaria phenotype [15]. Trautmann et al. suggested that premedication with antihistamines (5 mg desloratadine) 30 min before intake of a strong COX-I inhibitor may be an effective, safe, and practicable treatment in patients with NSAID-induced or NSAID-exacerbated urticaria [16].

Anand et al. [17] proved that inhibition of the COX2 inflammation pathway, in patients with chronic urticaria, may be clinically effective. A reduction of clinical symptoms and reduced demand for corticosteroids was observed in 5 of 8 refractory chronic idiopathic urticaria patients treated with antihistamines, antileukotrienes and corticosteroids, after adding rofecoxib (25 mg p.o.) to the therapy. The pathogenesis of urticaria is complex and not only related to histamine action. A more insightful understanding of the alternative pathways associated with urticaria may allow more effective treatments to be identified in patients with resistant urticaria phenotype. Well-designed studies on a larger group of patients need to be conducted to evaluate the role of lipid mediators in chronic autoimmune urticaria.

\section{Conclusions}

The effectiveness of levocetirizine and montelukast in chronic urticaria may be partly related to the changes in the serum level and the tissue expression of COX-1 and COX-2. These changes probably precede the clinical improvement, but further studies on a larger group of patients are needed to support this observation.

\section{Acknowledgments}

The study was financed by the Polish National Science Centre (NCN) UMO-2011/01/N/NZ5/03800.

\section{Conflict of interest}

The authors declare no conflict of interest.

\section{References}

1. Raciborski F, Kłak A, Czarnecka-Operacz M, et al. Epidemiology of urticaria in Poland - nationally representative survey results. Adv Dermatol Allergol 2018; 35: 67-73.

2. Kaplan A, Greaves MW. Urticaria and Angioedema. $2^{\text {nd }}$ edition. Informa Healthcare 2009.

3. Konstantinou GN, Asero R, Ferrer M, et al. EAACI taskforce position paper: evidence for autoimmune urticaria and proposal for defining diagnostic criteria. Allergy 2013; 68: 27-36.

4. Kupryś-Lipińska I, Kuna P. Efektywność terapii omalizumabem w przewlekłej pokrzywce spontanicznej. Alergol Współcz 2014; 32: 37-42.

5. Grattan CEH. Aetiopathogenesis of urticaria. In: Urticaria and Angioedema. Zuberbier T, et al. (eds.). Springer Verlag, Berlin Heidelberg 2010.

6. Smith WL, Garavito RM, DeWitt DL. Prostaglandin endoperoxide $\mathrm{H}$ synthases (cyclooxygenases)-1 and -2. J Biol Chem 1996; 271: 33157-60.

7. Kapp A, Pichler WJ. Levocetirizine is an effective treatment in patients suffering from chronic idiopathic urticaria: a randomized, double blind, placebo-controlled, parallel, multicenter study. Int J Dermatol 2006; 45: 469-74.

8. Potter PC, Kapp A, Maurer M, et al. Comparison of the efficacy of levocetirizine $5 \mathrm{mg}$ and desloratadine $5 \mathrm{mg}$ in chronic idiopathic urticaria patients. Allergy 2009; 64: 596-604.

9. Erbagci Z. The leukotriene receptor antagonist montelukast in the treatment of chronic idiopathic urticaria: a singleblind, placebo-controlled, crossover clinical study. J Allergy Clin Immunol 2002; 110: 484-8.

10. Di Lorenzo G, Pacor ML, Mansueto P, et al. Randomized placebo-controlled trial comparing desloratadine and montelukast in monotherapy and desloratadine plus montelukast in combined therapy for chronic idiopathic urticaria. J Allergy Clin Immunol 2004; 114: 619-25.

11. Thomson L, Blaylock MG, Sexton DW, et al. Cetirizine and levocetirizine inhibit eotaxin-induced eosinophil transendothelial migration through human dermal or lung microvascular endothelial cells. Clin Exp Allergy 2002; 32: 1187-92.

12. Ciprandi G, Cirillo I, Vizzaccaro A, et al. Levocetirizine improves nasal obstruction and modulates cytokine pattern in patients with seasonal allergic rhinitis: a pilot study. Clin Exp Allergy 2004; 34: 958-64.

13. Caproni M, Volpi W, Giomi B, et al. Cellular adhesion molecules in chronic urticaria: modulation of serum levels oc- 
curs during levocetirizine treatment. Br J Dermatol 2006; 155: $1270-4$.

14. Schäper C, Noga O, Koch B, et al. Anti-inflammatory properties of montelukast, a leukotriene receptor antagonist in patients with asthma and nasal polyposis. I Investig Allergol Clin Immunol 2011; 21: 51-8.

15. Pacor ML, Di Lorenzo G, Corrocher R. Efficacy of leukotriene receptor antagonist in chronic urticaria. A double-blind, placebo-controlled comparison of treatment with montelukast and cetirizine in patients with chronic urticaria with intolerance to food additive and/or acetylsalicylic acid. Clin Exp Allergy 2001; 31: 1607-14.

16. Trautmann A, Anders D, Stoevesandt J. H1-antihistamine premedication in NSAID-associated urticaria. J Allergy Clin Immunol Pract 2016; 4: 2005-12.

17. Anand MK, Nelson HS, Dreskin SC. A possible role for cyclooxygenase 2 inhibitors in the treatment of chronic urticaria. J Allergy Clin Immunol 2003; 111: 1133-6. 